Themenheft Nr. 44: Datengetriebene Schule.

Forschungsperspektiven im Anschluss an den 27. Kongress der DGfE

Herausgegeben von Mandy Schiefner-Rohs, Sandra Hofhues und Andreas Breiter

\title{
Zur (ambivalenten) Wirkmächtigkeit datengetriebener Lernplattformen
}

\author{
Eine Analyse des «Antolin»-Leseförderungsprogramms \\ Annina Förschler ${ }^{1}$ (D), Sigrid Hartong ${ }^{1}$ (D), Anouschka Kramer ${ }^{1}$, Claudia Meister-Scheytt ${ }^{1}$ \\ und Jaromir Junne ${ }^{1}$ (D) \\ ${ }^{1}$ Helmut-Schmidt-Universität Hamburg
}

\section{Zusammenfassung}

Mit einer Nutzung in über 85\% der Grundschulen zählt Antolin zu den in Deutschland beliebtesten digitalen Lernplattformen zur Förderung von Lesekompetenz. Auch weil Antolin hierbei in zunehmendem Masse direkt in schulweite digitale Infrastrukturen (Schulinformationssysteme) integriert wird, kann es als wirkmächtiges Element der wachsenden «Datengetriebenheit» von Schule verstanden werden, wurde aber bislang kaum als solches kritisch reflektiert. Diese Forschungslücke möchte der vorliegende Beitrag schliessen, indem er die Plattform als Erzeugung einer designbasierten, datafizierten und datafizierenden Form digitaler Bildungswelt in den Blick nimmt. Während die Plattform dabei einerseits erst aus dem vermittelten Zusammenspiel unterschiedlichster Praktiken (von Buchverlagen, Lehrpersonen, Schülerinnen und Schülern etc.) hervorgebracht wird, so erzeugt sie andererseits ein relationales, von Daten und Algorithmen geprägtes Gesamtgefüge, welches die genannten Praktiken auf spezifische Weise (un-)sichtbar, (un-)bedeutsam, (un-)möglich und unterschiedlich wertvoll macht. Gleichwohl wird Antolin je nach Kontext sehr unterschiedlich adaptiert, justiert und an bestehende Praktiken angeschlossen, sodass sich hier wiederum eine Vielzahl möglicher «Wirkungen» entfalten. Insgesamt, so soll dieser Beitrag zeigen, ist es dieses spezifische Zusammenspiel von Regulierung und kontinuierlicher Kontextualisierung, welches in Zukunft sowohl konzeptuell-methodisch als auch empirisch besser greifbar gemacht werden muss, um eine kritisch-bewusste Einschätzung und Gestaltung digitaler Lern- bzw. Datenplattformen als Kernmechanismus zunehmend «datengetriebener» Schule zu ermöglichen. 
On the Performativity of Data-Driven Learning Platforms. An Analysis of the «Antolin» Literacy Program

\begin{abstract}
Used in over 85\% of German elementary schools, Antolin has evolved into one of the most popular digital learning platforms for promoting reading skills. Also, because it is increasingly integrated into school-wide digital infrastructures (school information systems), it can be regarded as powerfully contributing to the ongoing datafication of schooling. However, critical reflections on Antolin have remained scarce. With this contribution, we aim to close this research gap by reconstructing the platform as a designbased, datafied and datafying form of digital education. While it is enacted through the mediated interaction of different practices (of book publishers, teachers, students etc.), the platform creates a relational, data-and algorithm-based infrastructure, which makes these practices (in-)visible, (in-)significant, (im-)possible and (un-)valued in a specific way. This does not mean, however, that Antolin is not adapted, adjusted and very differently contextualized, thus again unfolding various «effects». As this contribution will show, it is this specific interplay of regulation and continuous contextualization that scholars need to better understand - conceptually, methodologically, and empirically -, in order to enable a critical understanding of platforms as a central feature of the "data-driven» school.
\end{abstract}

\title{
1. Lücken in der (kritischen) Betrachtung datengetriebener Lernplattformen: Das Beispiel Antolin
}

Mit einer Nutzung in über $85 \%$ der Grundschulen und von über 5 Millionen Schülerinnen und Schülern (Stand Anfang 2020, ANT 9) zählte Antolin (www.antolin.de) bereits vor der Pandemie zu den in Deutschland beliebtesten digitalen Lernplattformen zur Förderung von Lesekompetenz, insbesondere seit der gezielten bildungspolitischen Hinwendung zur Förderung «digitaler Bildung» (BMBF 2016; KMK 2016; Westermann 2020a). Die Grundlogik der Plattform ist dabei auf den ersten Blick relativ unspektakulär: Nach Registrierung ganzer Klassen durch die Lehrperson lesen Schülerinnen und Schüler analog - meist nach der Schule zu Hause - selbst ausgewählte Bücher, für die auf der Plattform ein entsprechendes Quiz hinterlegt ist. Das Quiz wird nach dem Lesen bearbeitet, für richtige Antworten gibt es Punkte. Eine gezeichnete Rabenfigur mit Narrenkappe (= Antolin) begleitet das Quiz. Die Lehrperson erhält nach Abschluss des Quiz Datenauswertungen zur Quizleistung ihrer Schülerinnen und Schüler und kann dieses Wissen in ihren Unterricht einbeziehen (Abb. 1). 


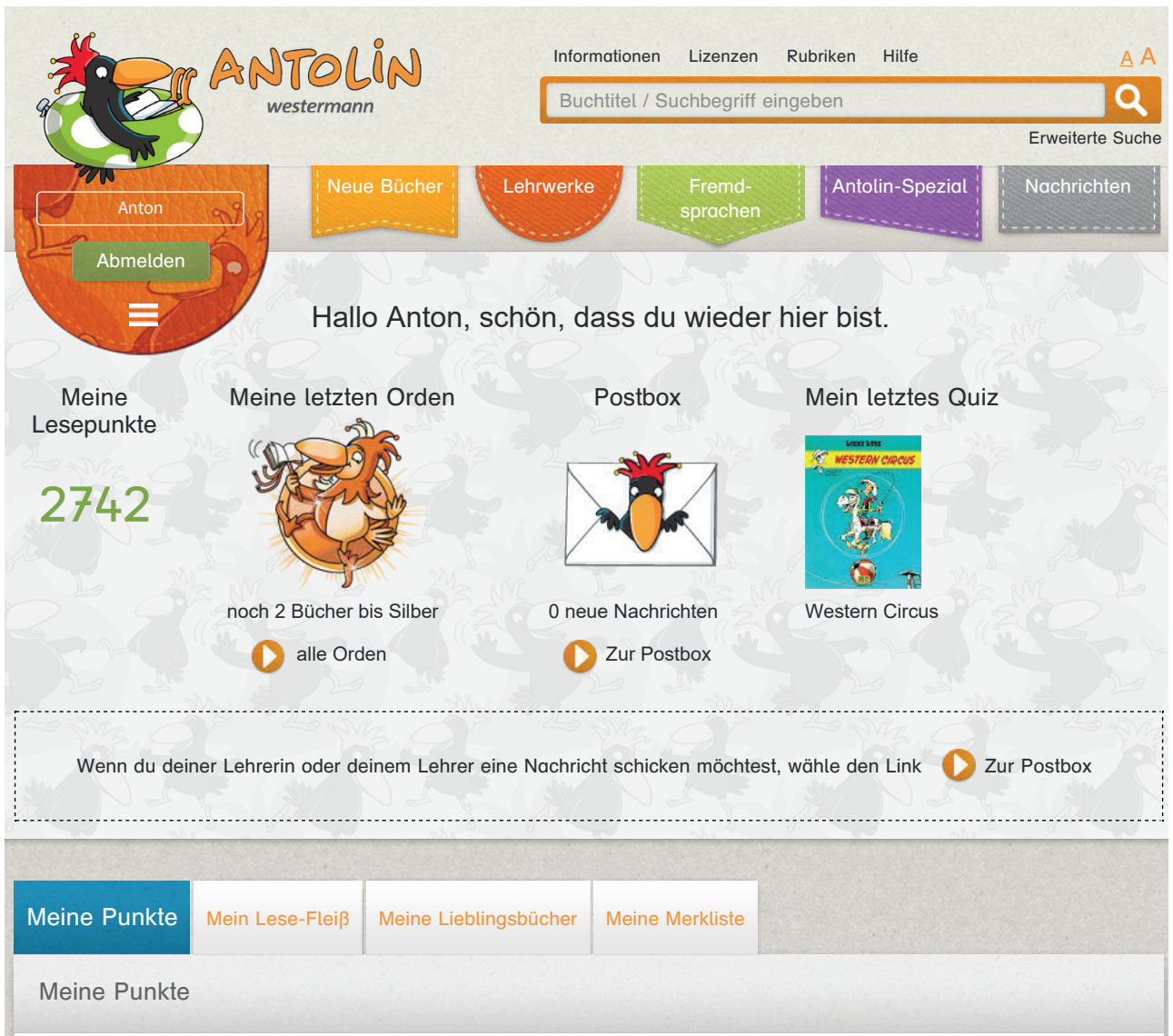

Abb. 1.: Screenshot Überblick «www.antolin.de, Schülerzugang» (Westermann 2020b, 4).

Zusammen, so bewirbt der Verlag die Plattform, böte Antolin damit ein viel versprechendes Werkzeug individualisiert-differenzierter Bildung, das gleichzeitig aufgrund des spielerischen Designs den Spass am Lesen und damit die Leistungsmotivation steigere sowie schliesslich den Lehrpersonen «hilfreiche Auswertungsinstrumente» in Form von Lesedaten-Profilen bereitstelle (Westermann 2020c, 4).

Insbesondere vor dem Hintergrund seiner enormen Verbreitung ist überraschend, dass es bislang nur sehr wenige kritische Studien zu Antolin gibt (v. a. Jornitz und Leser 2018; Viertel, Ehrenspeck-Kolasa, und Spies 2017). In den meisten anderen Materialien (z. B. Hochschulabschlussarbeiten, Evaluationen oder Praxisberichte aus Schulen, z. B. Henningsen 2019; Endlein 2009; Kleiner 2009; Westermann o.J.a) geht es hingegen beinahe ausschliesslich um die Frage, ob bzw. wie Antolin als funktionales Lernwerkzeug «tatsächlich» zu einer Motivations- und/oder Lesekompetenzsteigerung führt. 
Bewusst möchten wir einer solchen funktionalen Wirkungsperspektive in diesem Beitrag ein alternatives Verständnis entgegenstellen, welches die Wirkungen von Antolin vor allem in der Erzeugung einer designbasierten, datafizierten und datafizierenden Form digitaler Bildungswelt (vgl. Decuypere 2019, 416) sieht. Während die Antolin-Plattform dabei einerseits erst aus dem vermittelten Zusammenspiel unterschiedlichster Praktiken (von Buchverlagen, Lehrpersonen, Schülerinnen und Schülern etc.) hervorgebracht wird, so erzeugt sie andererseits ein relationales, von Daten und Algorithmen geprägtes (= datafiziertes) Gesamtgefüge, welches die genannten Praktiken auf spezifische Weise aus dieser Datenlogik heraus reguliert (= datafizierend) (Abschnitt 2, siehe auch Perrotta 2020; Hartong 2020). Diese wirkmächtige Regulierung von Bildung, Praktiken und Beziehungen möchten wir mit diesem Beitrag herausarbeiten (Abschnitt 3), gleichzeitig möchten wir auch zeigen, dass diese Regulierung keine Determinierung bedeutet (siehe hierzu auch Allert und Richter 2020). Vielmehr wird Antolin in verschiedenen Kontexten sehr unterschiedlich adaptiert, justiert und an bestehende Praktiken angeschlossen, sodass sich hier wiederum eine Vielzahl möglicher Wirkungen entfaltet (Abschnitt 4).

Insgesamt, so argumentieren wir, ist es dieses spezifische Zusammenspiel von Regulierung und kontinuierlicher Kontextualisierung, welches in Zukunft sowohl konzeptuell-methodisch als auch empirisch besser greifbar gemacht werden muss, um eine kritisch-bewusste Einschätzung und Gestaltung digitaler Lern- bzw. Datenplattformen als Kernmechanismus zunehmend «datengetriebener» Schule zu ermöglichen (Abschnitt 5).

\section{Lernplattformen als «Denkinfrastruktur»-konzeptuell-methodologische \\ Perspektiven auf einen zentralen Gegenstand datengetriebener Bildung}

Bereits seit einigen Jahren setzen sich Forscherinnen und Forscher aus (Medien-) Pädagogik, Kommunikationswissenschaft, Informatik oder Techniksoziologie im deutschsprachigen Kontext kritisch mit der zunehmenden Wirkmächtigkeit von Code, Software oder Big Data auf Bildung und Gesellschaft auseinander (z. B. Zorn 2011; Jörissen und Verständig 2017; Aßmann et al. 2017; Friedrichs-Liesenkötter et al. 2020). Eine dezidiertere Beschäftigung mit Konzepten wie Datafizierung, Plattformisierung, Automatisierung oder algorithmenbasierten Designs, die über vereinzelte Beiträge hinausgeht, findet sich hingegen erst seit relativ kurzer Zeit (z. B. Allert 2020; Jörissen 2018; Fischer 2019; Hartong und Förschler 2019; Jarke und Breiter 2019; Iske et al. 2020). Auch um derartige Perspektiven in den kommenden Jahren weiterzuentwickeln, argumentiert dieser Beitrag dafür, Befunde der sich international sukzessive etablierenden critical data bzw. critical platform studies systematisch aufzugreifen (z. B. Bucher 2018; Kitchin und Lauriault 2014; Iliadis und Russo 2016; Landri 2018; Williamson 2017; Decuypere und Landri 2020). Auch in diesen Studien geht es 
gezielt darum, gängige Vorstellungen - wie etwa, dass datenbasierte gleichbedeutend mit objektiviert-neutralisierter Steuerung sei oder (z. B. Lern-)Plattformen Orte der Öffnung/Teilhabe (vgl. Gillespie 2010, 350ff.) bzw. der instrumentell-neutralen Nutzbarmachung von Daten (Daten auf einen Blick) seien - bewusst zu hinterfragen. Stattdessen wird das Augenmerk auf die Macht von Daten und Plattformen gelegt und darauf, diese analytisch greifbar zu machen - sei es im Rahmen von schulischen Verwaltungssystemen (z. B. Breiter und Lange 2019), beim Monitoring von Schulen in der Schulsteuerung (z. B. Hartong und Förschler 2019; Ratner und Gad 2019; Landri 2018) oder im Rahmen der praktischen Unterrichtsgestaltung (z. B. Manolev, Sullivan, und Slee 2019). ${ }^{1}$

Im Anschluss an diese Forschung verstehen wir jede Plattform als Konstruktion einer designbasierten, datafizierten und gleichzeitig datafizierenden Form digitaler Welt bzw., im Fall von Lernplattformen, digitaler Bildung (vgl. Decuypere 2019, 416): Jedes auf der Plattform auf bestimmte Art und Weise (nicht) sichtbare oder (nicht) bearbeitbare Element (im Falle von Antolin z. B. Schülerinnen und Schüler, Bücher, Quiz etc.) ist das Ergebnis zahlreicher (politischer) Momente der Selektion, der Modellierung und «Inwertsetzung» (Hartong 2020; Bowker et al. 2019, 4; Ruppert, Isin, und Bigo 2017), und damit im Design eingeschriebener normativer Vorstellungen von «gutem» Lesen, "guter» Lernentwicklung etc. Diese werden über unterschiedliche Mechanismen und oftmals unbewusst an die Nutzerinnen und Nutzer vermittelt und ihr Handeln/Entscheiden entweder bei der unmittelbaren Interaktion mit der Plattform (on and with the platform) oder aber bei der mittelbaren Interaktion untereinander mit Bezug auf die Plattform (beyond the platform) reguliert (vgl. Decuypere 2021, siehe auch unten). Derartige Mechanismen der Regulierung umfassen etwa die Standardeinstellungen, die strategische Nutzerführung durch die Plattformfunktionen oder die konkrete Modellierung dessen, was im Design wie in Daten übersetzt wird.

Mit Bowker und Kollegen und Kolleginnen (2019) kann Antolin (bzw. können Plattformen in der Bildung insgesamt) damit als eine Art «Denkinfrastruktur» (thinking infrastructure) erfasst werden, welche die Autoren und Autorinnen definieren als «[...] form of distributed cognition and distributed agency that structure collective reasoning, attention and decision-making across multiple sites» (ebd., 5). Mit dem Konzept wird einerseits betont, dass Plattformen nicht technologisch-objektiviert für sich existieren (= Werkzeug, siehe auch Jornitz und Leser 2018, 56). Vielmehr werden sie (erst) aus dem vermittelten Zusammenspiel unterschiedlichster Praktiken dynamisch und immer wieder neu hervorgebracht. Gleichzeitig tritt keine Plattform als «ein Ganzes» oder als «flache Oberfläche» in Erscheinung, sondern immer in Form von Modulen einer kompositionell verschachtelten Architektur (vgl. Decuypere 2021) - im Falle von Antolin z. B. der Quizbereich, der Bereich der Interaktion mit Mitschülerinnen und Mitschülern, die Datendashboards, aber ebenso die Integration

1 Siehe hierzu auch die aktuellen BMBF-Verbundprojekte www.datafied.de sowie https://all-is-data.de/. 
von Antolin als Plattform in übergreifende Schulinformationssysteme. ${ }^{2}$ Andererseits bringen Plattformen dieses dynamische, modularisierte Zusammenspiel, welches oftmals mit der Idee von Flexibilisierung und Personalisierung verwechselt wird, in ein relationales Gesamtgefüge, welches die Praktiken, die Beziehungen zwischen Lehrpersonen, Schülerinnen und Schülern, Eltern etc. sowie Möglichkeiten des Denkens (vgl. Bowker et al. 2019, 2) reguliert. Diese Regulierung bezieht sich nicht nur auf die kognitive Ebene, sondern ebenso auf die emotionale Wahrnehmung bzw. auf die Ebene möglicher Identitätsbildung und Subjektivierung durch die Interaktion mit der Plattform (ebd., 7f.).

Insgesamt ergibt sich die Wirkmächtigkeit von Plattformen als Denkinfrastrukturen also aus dem komplexen Zusammenspiel zwischen stabilisierender Regulierung einerseits und kontinuierlicher Dynamik andererseits (vgl. Martin und Secor 2014, 422). D. h., bei aller Regulation und damit Einschränkung dessen, was möglich ist, manifestieren sich nach wie vor vielfältige (ggf. auch widersprüchliche) Momente der Kontextualisierung, je nachdem, von wem die Plattform in welchem Setting wie genau genutzt und welcher Sinn ihr damit zugeschrieben wird (vgl. Perotta 2020, 5; Ratner und Gad 2019, 539; Reich 2020). ${ }^{3}$

Methodologisch bringt eine solche relationale Perspektive auf die dynamische, stets kontextualisierte Wirkmächtigkeit von Plattformen zweifellos neuartige Herausforderungen mit sich, insbesondere, was konkrete methodische Zugänge angeht (vgl. Gulson et al. 2017). Für diesen Beitrag greifen wir entsprechend auf aktuelle Ansätze der methodischen Systematisierung in den critical platform studies zurück, in denen zwischen mehreren Einstiegstüren zur möglichen Beforschung von Plattformen unterschieden wird (vgl. Decuypere 2021). Konkret kombinieren wir hierbei drei analytisch-methodische Perspektiven: (1) Die Oberfläche von Antolin (= on the platform), (2) direkte Nutzungspraktiken von Antolin durch Lehrpersonen, ihren Schülerinnen und Schülern sowie Eltern (= with the platform) sowie (3) Praktiken mit indirektem Bezug zu Antolin, die auf den breiteren Kontext der situativen Plattformumgebung hinweisen (= beyond the platform).

In Bezug auf die Antolin-Oberfläche dokumentierten wir über die Erstellung von Screenshots und Notizen das Arrangement und damit die Regulierungskraft der Plattform. Dieses Arrangement umfasst die unterschiedlichen Funktionen, Module und Sichtbarkeiten (als Kombination von Text- und Bildelementen), die insgesamt die durch Design, Daten und Algorithmen erzeugte «Umgebung der erwarteten Nutzung» (environment of expected use; Decuypere 2021; vgl. Dieter et al. 2018, 5) bzw. die Denkinfrastruktur erzeugen. Hierbei wurde sowohl aus der Perspektive von

2 So wird Antolin zunehmend als eine Art schnittstellenbasiertes Modul in übergreifende Schulinformationssysteme integriert - etwa itslearning (https://itslearning.com/de/), welches in Bremen und SchleswigHolstein inzwischen sogar als landesweit vereinheitlichtes Lernmanagementsystem genutzt wird.

3 Siehe zu dieser Dimension vielfältiger Praktiken des medialen enactment auch klassischere Beiträge der Medienforschung (z. B. Ang 1995). 
Schülerinnen und Schülern als auch derjenigen von Lehrpersonen auf die Plattform zugegriffen, um zu analysieren, wie jeweils beide Gruppen die Plattform «durchlaufen», was sie wann auf der Plattform (nicht) tun können bzw. sollen, was sie an welcher Stelle wie zu sehen bekommen, wo normative Einschreibungen sichtbar werden (z. B. Vorstellungen von «gutem» Lesen), aber ebenso, an welchen Stellen sich Möglichkeiten der Gestaltbarkeit manifestieren. Ergänzend wurden Beschreibungen der Plattformoberfläche auf der Antolin-Homepage sowie in online zugänglichen WerbeMaterialien des Verlags hinzugezogen und analog ausgewertet. Das Hinzuziehen dieser weiteren Materialien war insbesondere nötig, um Wechselwirkungen von Partizipation und Regulierung im Rahmen der Produktion bzw. des (immer wieder neuen) «Hervorbringens» von Antolin (z. B. bzgl. der Erstellung von Quiz oder des Designens der Buchauswahl) erfassen zu können (siehe Abschnitt 3).

Die Rekonstruktion der direkten und indirekten Nutzungspraktiken bzw. der Kontextualisierung von Antolin basiert wiederum zum einen auf insgesamt 14 leitfadengestützten Interviews mit Lehrpersonen, Eltern sowie der Produzierendenseite (ANT 1-14). Die Leitfragen befassten sich zum einen sowohl mit der eigenen als auch mit der bei anderen beobachteten Nutzung (z. B. Lehrpersonen berichteten über ihre eigene Nutzung, über die Nutzung durch die Schülerinnen und Schüler sowie Eltern ihrer Klasse) und schliesslich mit der persönlichen Einschätzung und Reflexion der Wirkung(-en) von Antolin. Zum anderen sammelten wir insgesamt 154 Beiträge auf zwei bekannten Eltern-Onlineforen. Beides wurde induktiv codiert, indem Themen sowie wiederkehrende Schlagworte identifiziert, geclustert, gruppiert und nach Häufigkeit ausgewertet wurden. Aus dieser Auswertung heraus wurden in einem nächsten Schritt sowohl Typen generiert (etwa Typen von Eltern bzgl. ihrer Nutzung von Antolin) als auch unterschiedliche Dimensionen der Kontextualisierung der Plattform (siehe Abschnitt 4).

Wenngleich wir mit unserer Analyse keine Gesamteinschätzung der Wirkmächtigkeit von Antolin geben können, ermöglicht diese methodische Kombination unterschiedlicher Perspektiven dennoch eine Reihe von Anknüpfungspotenzialen für weitergehende Analysen und Diskussionen.

\section{Wirkmächtigkeit by design: Einblicke in die Antolin-Oberfläche (on the platform)}

Anhand zweier Fragen - Was ist ein «Buch»? (3.1) sowie Was ist «Lesen»? (3.2) - möchten wir in diesem Abschnitt beispielhaft zeigen, wie die Oberfläche von Antolin als Denkinfrastruktur auf der einen Seite durch vielfältige Praktiken unterschiedlicher Akteure hervorgebracht, deren Zusammenspiel aber andererseits in eine wirkmächtige Form gebracht und reguliert wird. Ausgangspunkt für die Beantwortung beider Fragen ist dabei, dass Antolin keine Leseplattform ist, das Lesen von Büchern selbst 
also nicht auf der Plattform stattfindet. Dennoch manifestiert sich das analoge Lesen von analogen Büchern als zentraler Referenzpunkt von Antolin, während das Design der Plattform - und dies ist der zentrale Gedanke des Denkinfrastrukturkonzepts - diesen analogen Referenzpunkt in eine Reihe weiterer (digitaler) Referenzpunkte einordnet, inn entlang des Designs formt und auf bestimmte Weise sichtbar macht. Durch diese Sichtbarkeit werden Bücher und Lesen wiederum erst denk- und bearbeitbar. Im Umkehrschluss bedeutet dies aber auch, dass Bücher und Lesen, die nicht dieser Form entsprechen, im Rahmen der Denkinfrastruktur von Antolin nicht sicht-, denk- und bearbeitbar werden.

\subsection{Was ist ein «Buch»?}

Auf seiner Oberfläche wirbt Antolin mit einer riesigen «Buchauswahl», die etwa Klassiker und Neuerscheinungen der Kinder- und Jugendliteratur, Belletristik, Sachbücher, Bücher in einfacher Sprache, Gedichte, Comics, Nachrichten, Lehrwerks- und Gebrauchstexte und sogar fremdsprachige Bücher umfasst und aus der, so der Verlag, frei gewählt werden kann. Formell partizipieren also eine Menge unterschiedlicher Verlage an der gemeinsamen «Hervorbringung» von Antolin (von Bowker et al. 2019 als distributed agency beschrieben, siehe Abschnitt 2), allerdings nur, sofern ihre Bücher eine ISBN-Nummer aufweisen (ANT 9) - dasselbe Kriterium, welches in der analogen Welt üblicherweise gilt, um Bücher als erwerbbare Bücher zu klassifizieren.

Aus Perspektive der Denkinfrastruktur rückt jedoch noch ein anderer Mechanismus in den Fokus, der reguliert, ob und wie «ein Buch» auf Antolin denk-, sicht- und bearbeitbar wird: die im Design verankerte Kopplung von Buch, Quiz und «Narrenkappe»: So markiert das Absolvieren eines Quiz als notwendige Grundlage für die Produktion von Lesepunkten (= Daten über Lesen, siehe unten) den stärksten «obligatorischen Durchgangspunkt» (obligatory point of passage, Decuypere 2019) der Plattform bzw. den Kern der gesamten Plattformmodellierung. Dies bedeutet, dass sämtliche an der Plattform partizipierenden Akteurinnen und Akteure das Element Quiz gleichermassen «passieren» müssen. Auch Bücher müssen im Sinne dieser Logik unmittelbar an ein Quiz gekoppelt sein, und zwar als deren «Vorschaltung»; ohne diese Relationierung werden sie auf der Plattform nicht sichtbar. ${ }^{4}$

Die Quiz werden dabei nicht (nur) in Eigenregie vom Westermann Verlag entwickelt, sondern von unterschiedlichen Quizdesignerinnen und -designern - zu circa 70 Prozent von engagierten Lehrpersonen und Eltern, selten auch von Autorinnenund Autorenteams anderer Verlage zu ihren jeweiligen Büchern -, die also auch hier (wohlgemerkt ohne Bezahlung) an der Generierung zentraler Inhalte der Plattform partizipieren. ${ }^{5}$ Jedoch muss jede Quizeinreichung eine bestimmte Form haben (etwa

4 Heraus fallen hierbei vor allem «Nischenbücher» und Bücher kleiner unbekannter Verlage (ANT 9/10).

5 Stand April 2020 weist Antolin insgesamt fast 100.000 generierte Quizsätze auf (ANT 9). 
im Multiple-Choice-Format vorliegen); sie wird vom Verlag begutachtet und nur eine «qualitativ gesicherte» Auswahl gelangt in Kopplung mit Büchern auf die Plattformoberfläche.

Die übergreifende Logik dieser Kopplung basiert hierbei auf dem System der Narrenkappe, die der auf der Plattform aktive Antolin-Rabe nicht nur symbolhaft auf dem Kopf trägt, sondern die auch die zentrale Passage der Inwertsetzung im Design markiert. Denn über die Kappenfarbe - rot, blau oder grün - werden Quiz zu einem Buch einem von drei Schwierigkeitsleveln zugeordnet, was entscheidend dafür ist, wie viele Fragen welcher Art ein Quiz enthält und wie viele Punkte durch das Quiz erzielt werden können: «Je leichter zu lesen und zu verstehen ein Kinderbuch ist, desto weniger Punkte gibt es» (Zschocher 2020; vgl. Westermann o.J.b). Dies hat gleichzeitig unmittelbare Konsequenzen darauf, wie Lesen bzw. Lesende reguliert werden (siehe Abschnitt 3.2).

Gerahmt durch diese übergreifende Inwertsetzung, die sich aus der Logik der Datafizierung im Sinne der Notwendigkeit quantifizier- und hierarchisierbarer Datenwerte (Punkte) ergibt, kreiert die Plattform wiederum weitere modularisierte Ordnungen von Sichtbarkeit. Diese betreffen insbesondere die Suche nach Büchern als Quiz-Optionen. So gibt es auch hier auf der einen Seite eine Unmenge von (untereinander kombinierbaren) Suchmöglichkeiten, die auf der anderen Seite jedoch stets im Rahmen der Grenzen dessen verbleiben, was im Design als kategoriale Grund- und Sortierprinzipien festgeschrieben ist. Antolin unterteilt nicht in Buchgenres, sondern setzt zum einen auf eine Freitextsuche mit automatisierten Vorschlägen (vergleichbar mit dem Operieren der Google-Suchmaschine), zum anderen auf einen Kategorienmix aus z. B. Neuerscheinungen sowie vom Verlag zusammengestellten Buchvorschlägen. Neben Lesetipps in der Kategorie «Neue Bücher» gibt es zudem den Reiter «TOP 100» (generiert aus diversen Datenpunkten ${ }^{6}$ ).

Die jeweiligen Suchergebnisse lassen sich wiederum sortieren - insbesondere nach «Beliebtheit», «Beliebtheit bei Jungen» und «Beliebtheit bei Mädchen». Diese Beliebtheitsfilter basieren auf Sternebewertungen (1-5 Sterne - «Wie hat dir das Buch gefallen?»), die die Nutzerinnen und Nutzer vor einer Quizdurchführung abgeben und damit wiederum an der Gestaltung der Buchsortierung partizipieren können. Gleichzeitig erfolgt diese Partizipation ausschliesslich über das datafiziertquantifizierte Format der Sterne sowie - an dieser Stelle erneut ein zentrales Moment der Inwertsetzung - geteilt in Jungen und Mädchen, was bedeutet, dass das Design hier einen binär geschlechtstypischen, systematischen Unterschied der Lesevorlieben und der Präferenzsetzung (re-)produziert.

6 Hierfür wird ausgewertet, welche Bücher am häufigsten ausgewählt und welche Quiz am häufigsten absolviert werden, differenziert nach Grundschule, Sekundarstufe sowie verschiedenen Zeiträumen (vgl. Westermann 2017, 30). 


\subsection{Was ist «Lesen»?}

Eng verbunden mit Büchern als zentralem Element der Antolin-Denkinfrastruktur ist die Frage, wie «Lesen» als (nicht) mögliche bzw. (nicht) gewollte Praxis auf der Plattformoberfläche erzeugt und reguliert wird. Auch hier wird die Positionierung der Quiz als Dreh- und Angelpunkt des Designs deutlich. So kann das analoge Lesen lediglich retrospektiv in Form quizbezogener Datenpunkte (Lesepunkte, Logdaten über die benötigte Zeit usw.) sicht-, denk- und bearbeitbar werden, während die Zahl der absolvierten Quiz automatisch mit der «Zahl der gelesenen Bücher» gleichgesetzt wird. Zwar werden in der Regel ja auch die Quizfragen gelesen; dieses Lesen ist aber nicht das, worauf es ankommt - was sich etwa auch darin zeigt, dass sich Schülerinnen und Schüler die Quizfragen sogar von der Plattform vorlesen lassen können. ${ }^{7}$

Die Fokussierung auf das Absolvieren des Quiz bedeutet gleichzeitig, dass Lesen auf der Plattformoberfläche zu einer Praxis wird, die entweder erfolgreich ist oder nicht, formiert als richtige oder falsche Antworten (= viele oder wenige Punkte ${ }^{8}$ ) sowie als benötigte Zeit, in der Regel gekoppelt mit einer Zeit- und Versuchslimitierung (= one try design, siehe hierzu auch Jornitz und Leser 2018,64$).{ }^{9}$ Gleichzeitig soll die Zeitrestriktion ein Unterlaufen der Vor-/Nachordnungslogik verhindern, also, dass während des Quiz im analogen Buch nachgelesen wird, anstatt das Quiz nach dem Lesen des Buches zu bearbeiten.

Zusätzlich finden sich im Rahmen der Quizabsolvierung eine Reihe von Designelementen (v. a. Elemente der Gamifizierung wie die Punkte- und Preisvergaben, der Antolin-Rabe als motivierender Avatar etc.), die auf die affektive, emotionale Dimension abstellen und damit auf die von Bowker et al. $(2019,7 \mathrm{f}$.) betonte Ebene möglicher Identitätsbildung bzw. Subjektivierung als (erfolgreiche/r) Leser/in (sculpted subjectivities; Decuypere 2019, 424). Durch diese Elemente wird zusätzlich sichergestellt, dass Nutzerinnen und Nutzer die im Design eingeschriebene normative Vorstellung von Lesen als richtige Antworten binnen kurzer Zeitdauer als die Betrachtung und Bewertung ihrer analogen Lesepraxis übernehmen.

Auch für die Frage «Was ist Lesen?» spielt die Inwertsetzungslogik der Narrenkappe eine entscheidende Rolle. So werden nicht nur Bücher und Quiz entlang der drei Schwierigkeitslevel geordnet und in Wert gesetzt, sondern ebenso die Lesepraxis als Quizpraxis, folglich die Nutzerinnen und Nutzer selbst. So steht die rote Kappe für «11 oder 16 Fragen zum Inhalt, zum Nachdenken, zum Bilden einer Meinung», die blaue Kappe für «10 oder 15 Fragen zum Inhalt» und die grüne Kappe für «10 einfache Fragen für Leseanfänger oder leseschwache Kinder» (Westermann o.J.b, o.S.). Mit der

7 Diese Funktion kann von Lehrpersonen ein- und abgeschaltet werden (Westermann 2017, 14).

8 Standardbasiert führen falsche Antworten in Antolin zu Minuspunkten, sodass das Gesamtquiz auch mit einem negativen Punktewert abgeschlossen werden kann. Diese Funktion kann aber von den Lehrpersonen ausgestellt werden (Westermann 2017, 14; 2020b, 2, 4, 6).

9 Auch die Zeitlimitierung kann von den Lehrpersonen ausgeschaltet werden. Zudem kann eine Lehrperson bestimmte Bearbeitungszeiten für die Absolvierung von Quiz festlegen (Westermann 2017, 14). 
Überführung von Lesen in dieses lineare Entwicklungsmodell dreier aufeinander aufbauender Stufen findet eine weitere signifikante Reduktion sichtbarer analoger Lesepraxis statt (siehe hierzu kritisch Viertel, Ehrenspreck-Kolasa, und Spies 2017 sowie Jornitz und Leser 2018), bei der beispielsweise Elemente wie Meinungsbildung von den ersten beiden Stufen exkludiert werden. Zugespitzt formuliert bedeutet dies, dass Meinungsbildungsmomente von Lesenden, die Quiz mit grüner Kappe durchführen, unsichtbar sind und damit tendenziell als wertlos erscheinen.

Am Ende werden die im Antolin-Design als relevant definierten Datenpunkte der Quizpraxis in Datendashboards überführt, wobei an dieser Stelle insbesondere die Lehrpersonen-Dashboards interessant sind. Als datenbasierte, zusammenfassende Einschätzung der «Leseleistung» von Schülerinnen und Schülern einer Gesamtklasse bilden sie eine verdichtete Form designbasierter Qualitätskriterien (vgl. Jornitz und Leser 2018, 69) und gleichzeitig einen erweiterten Regulierungsmoment bzgl. der analogen Unterrichtspraxis, sofern die Lehrperson die Datendashboards für deren Gestaltung - oder sogar für die Benotung ihrer Schülerinnen und Schüler - benutzt (siehe hierzu auch Abschnitt 4). Wie die Buchsuche lässt sich auch das Datendashboard «flexibel» anordnen; aber auch hier bewegt sich diese Flexibilität innerhalb vorgegebener Filter- und Sortieroptionen. Diese umfassen pro Kind die durchschnittliche Prozentzahl der möglichen erreichbaren Quizpunkte (Westermann 2017, 7), die durchschnittliche Punktzahl pro Woche oder Monat, die Anzahl der «gelesenen» Bücher (was bedeutet: die Anzahl der durchgeführten Quiz) pro Woche oder Monat sowie die durchschnittliche Quizzeit. Entlang jedes Kriteriums kann eine hierarchische Sortierung der Klassenliste angeordnet werden. Auch hier zeigt sich also, wie eine wirkmächtige Sichtbarkeitsregulierung im Sinne des möglichen Nachdenkens über und Reagierens auf «das Verhältnis der Schülerin und des Schülers zum Buch» (Jornitz und Leser 2018, 68 f.) auf Lehrpersonen «wirkt».

\section{Zwischen Regulierung und Kontextualisierung: Zur direkten und indirekten Nutzung von Antolin (with and beyond the platform)}

Im Anschluss an die beispielhaften Betrachtungen der regulierenden Wirkmächtigkeit der Plattformoberfläche von Antolin geht es in diesem Abschnitt darum, das Wechselspiel zwischen dieser Regulierung und Momenten der Kontextualisierung der Plattform im Kontext ihrer tatsächlichen Nutzung - im engeren (with the platform) und weiteren (beyond the platform) Sinne - zu betrachten.

Den Einstieg bildet hierbei eine überblicksartige Darstellung der von uns im Rahmen der Auswertung identifizierten Kontextualisierungsdimensionen (siehe Abb. 2, äusserer Ring), bevor wir einige dieser Dimensionen anhand ausgewählter Befunde aus den Lehrpersonen- und Elterninterviews sowie den Forenanalysen näher illustrieren. 


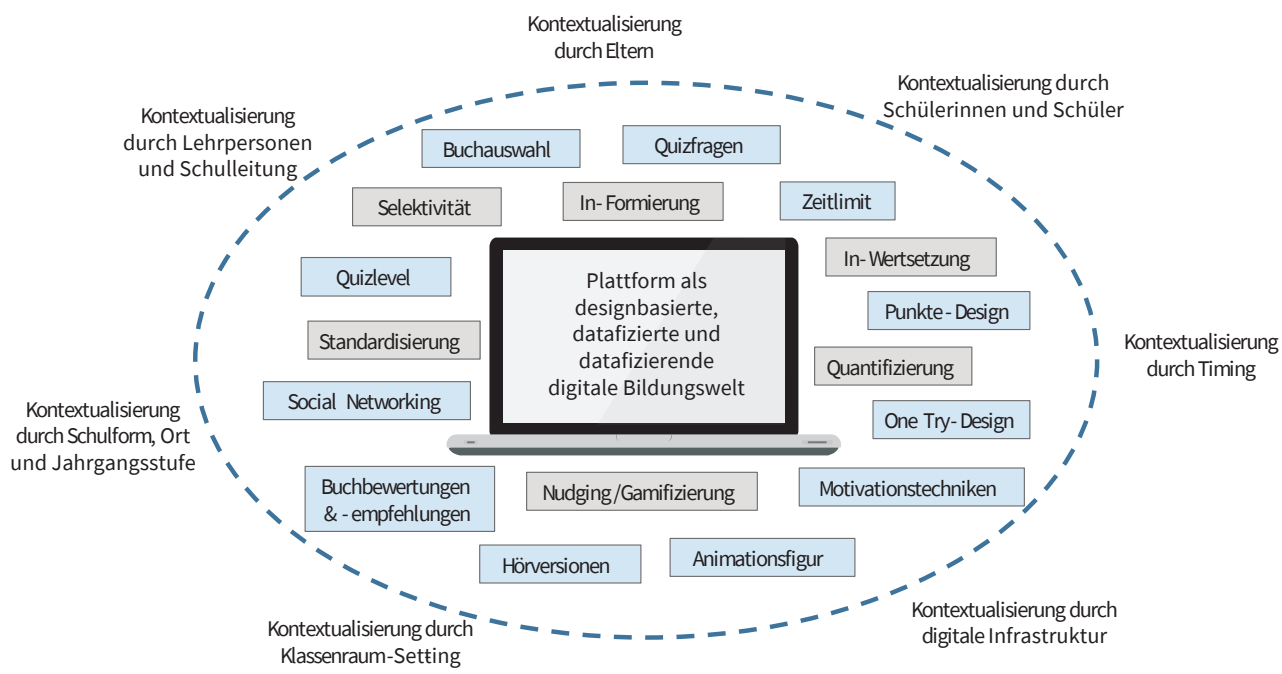

Abb. 2.: Antolin im Zusammenspiel aus Plattformsetting und Kontextualisierung (eigene Darstellung).

\section{1 «Ich hab das einfach mal eingeführt, weil ich dachte, es ist nett» (ANT 3) - was Lehrpersonen berichten}

In der Tat variiert die Nutzung von Antolin durch Lehrpersonen stark. Diese Variation bezieht sich zum einen auf die überhaupt genutzten Funktionen der Plattform: Wenngleich es durchaus Lehrpersonen gibt, die «besondere Module» von Antolin einsetzen (z. B. die Postbox, Ordensvergabe oder Lese-Fleiss - z. B. ANT 4), so nutzen die meisten Lehrpersonen hauptsächlich dessen Kernfunktion: das Beantworten von Quizfragen durch Schülerinnen und Schüler zu gelesenen Büchern. Hier zeigt sich entsprechend, was bereits Viertel, Ehrenspeck-Kolasa und Spies konstatieren, nämlich, dass «Funktionen und Instrumente [von Antolin, wie] [...] z. B. Leseanregungen, Arbeitsmaterialien oder die Möglichkeit, eigene Fragen zu erstellen [...], in der Praxis (fast) gar keine Rolle spielen» $(2017,161)$. Gleichzeitig verlassen sich die von uns interviewten Lehrpersonen zum Grossteil auf die voreingestellten StandardSettings der Plattform (z. B. Minuspunkte an, Zeitzähler an, Vorlesefunktion aus). Dabei fiel auf, dass selbst Wissen über grundlegende Standardfunktionen (z. B. «Kann man Fragen wiederholen?» oder «Gibt es ein Zeitlimit für Quizze?») und insbesondere hinsichtlich möglicher (im Rahmen des Designs individuell anpassbarer) SettingOptionen kaum vorhanden ist - trotz teilweise langjähriger Nutzung des Programms (ANT 3/4/13). ${ }^{10}$

Zum anderen beziehen sich die Variationen auf die Art und Weise der Nutzung. In den meisten Fällen wurde angegeben, Antolin auf freiwilliger Basis als «Add-On»

10 Infoseiten und Lehrpersonenhandbücher von Seiten des Verlags sind verfügbar (siehe z. B. Westermann 2017; o.J.d; o.J.e). 
(ANT 4) einzusetzen, um Schülerinnen und Schülern mehr "Spaß» am Lesen zu ermöglichen (ANT 3/4/14). Es gibt aber auch zahlreiche Lehrpersonen, die Antolin als 〈Messinstrument) nutzen und für die die statistischen Auswertungsfunktionen ein zentrales Element sind bzw. teilweise sogar in die Notengebung einfliessen (F1B2; F2B32/34; vgl. Viertel, Ehrenspeck-Kolasa und Spies 2017, 159). ${ }^{11}$ Hierbei wird die Erfassung des Leseumfangs oft als sehr zuverlässig eingeschätzt, da «nach vollkommen klaren Vorgaben bestimmte Punkte erreicht werden können oder nicht» (ANT 13; vgl. Kleiner 2009, 275). Es gibt aber auch zahlreiche kritischere Einschätzungen der Leseleistungserfassung (ANT 3/6/9/12/13/14). Dabei wird immer wieder auf Validitätsprobleme aufgrund von Manipulation verwiesen, da es angesichts des Arbeitens mit Antolin zu Hause keine gänzliche Kontrolle durch die Lehrperson gäbe. So berichten einige Lehrpersonen von zahlreichen «kreativen» Wegen, das System auszutricksen (ANT 3/13, siehe auch Abschnitt 4.2).

Kritisch betrachtet wird auch die Wirkung von Antolin auf den Unterricht sowie auf die Kommunikation über Lesen und Bücher. So ginge es laut Einschätzung einiger Lehrpersonen nicht mehr um «Lesen an sich» (ANT 6), um die Möglichkeit des Eintauchens in ein Buch oder den Austausch über Inhalte und Emotionen (ANT 14). Das Design der Plattform bewirke bei Schülerinnen und Schülern vielmehr eine Fokusverschiebung hin zum Punkteerwerb - es gehe schnell um «Punkte fressen» statt um Lesequalität (ANT 6/13). Entsprechend komme es zu kompetitiven Verhaltensmustern (z. B. Kämpfe um die Computernutzung in schulischen Antolin-Sessions; Kommentieren von Quizdurchläufen von Mitschülerinnen und -schülern etc.). Spannenderweise wurde dieser Effekt selbst dann beobachtet, wenn die Lehrperson Punktestände bewusst nicht in der Klasse thematisiert (ANT 13). Es gibt aber durchaus auch Lehrpersonen, die diesen Wettbewerb über Antolin explizit fördern, indem sie z. B. die Punktestände von Kindern vor der Klasse vorlesen, Orden vergeben oder Urkunden verteilen (ANT 12/14; F4B25; F2B26; F3B14).

Insgesamt weisen die Ergebnisse also zum einen auf die zentrale Rolle der Lehrperson als Plattform-Gatekeeper hin, was sich auch darin zeigt, dass überhaupt nur über Lehrpersonen Konten für Schülerinnen und Schüler angelegt werden können (Lizenzen für Privatpersonen wie Eltern gibt es nicht, vgl. Westermann o.J.c). Zum anderen wurde in den Auswertungen deutlich, dass die (mögliche) Nutzung durch die Einbettung in den schulischen Kontext stark vorgeprägt bzw. reguliert wurde. Wenn Antolin an einer Schule bereits intensiv oder gar obligatorisch eingesetzt wird, werden neu angestellte Lehrpersonen z. B. nahezu automatisch zu Nutzerinnen und Nutzern. Eine Lehrperson berichtete entsprechend davon, dass sie sich an ihrer Antolin-affinen Schule für ihre Nicht-Nutzung rechtfertigen musste und ihr

11 Damit wird auch wichtig, ob Bücher nur in der Schule (z. T. in eigenen «Antolin-Stunden»), auch zuhause, oder ausschliesslich zuhause gelesen und Quiz dementsprechend in der Schule oder zuhause gemacht werden (sollen). Diese Offenheit ist vom Verlag durchaus gewollt (vgl. Westermann o.J.d). 
«Technikverweigerung» vorgeworfen wurde (ANT 14). Genau gegenteilig schilderte eine andere Lehrperson ihre Überraschung über eine «Welle der Empörung» (ANT 3) von Seiten der Eltern, als sie Antolin (wie an ihrer vorherigen Schule gängig) einführen wollte. Auffallend war insgesamt, dass es für die meisten Interviewten an ihrer entsprechenden Schule keine pädagogische Einführung in die Plattform (Funktionen, Einstellungsoptionen, pädagogische Fragen) gab, was etwa damit begründet wurde, dass Antolin «einfach» und «selbsterklärend» sei (vgl. u. a. ANT 13/14).

Bezüglich der organisationalen Verankerungen gibt es an einigen Schulen direkte Schnittstellen zu Antolin über das jeweilige übergreifende Schulinformationssystem und entsprechende Schullizenzen, die alle Lehrpersonen nutzen können oder müssen. An einzelnen Schulen ist Antolin sogar curricular verankert (z. B. in festen «Antolin-Stunden» - vgl. Westermann o.J.d; ANT 3), es gibt sogenannte "Leseschulen» (Westermann o.J.e), «Antolin-Wochen» (ANT 14) oder Schulen, die öffentlich auf ihrer Homepage die «besten Antolinsammler» auszeichnen (Grundschule Hönebach o.J.). In anderen Schulen haben wiederum nur einzelne Lehrpersonen Klassenlizenzen und Antolin manifestiert sich entsprechend wesentlich unsystematischer. ${ }^{12}$

\section{2 "Auf dem Schulhof ist auch schon ein richtiger, Mein Kind hat aber mehr Punkte" Wettbewerb ausgebrochen» (F3B1) - was Eltern berichten}

Auch Eltern haben einen grossen Einfluss auf die Nutzung von Antolin. Grundlegend gilt: ohne ihre Zustimmung darf rein rechtlich (eigentlich) kein Konto für das Kind angelegt werden (ANT 13). ${ }^{13}$ Auch das Antolin-Design konstruiert Eltern als zentrale, jedoch indirekte (ohne eigene Zugangsmöglichkeiten) Partizipierende an der Plattform. So umfasst etwa die Antolin-Homepage eine eigene Seite zum Thema «Mitarbeit der Eltern", auf der beispielsweise betont wird, dass Eltern zum Lesen motivieren bzw. die Antolin-Bearbeitungszeit betreuen sollen (vgl. Westermann o.J.f). Die zentrale Rolle der Eltern wurde auch in unseren Interviews und den Forenbeiträgen thematisiert und bestätigt (vgl. z. B. ANT 11/4; F4B22). Es zeigte sich jedoch auch, dass Eltern keinen wirklich «aktiven Moment der Zustimmung» erlebt und selten Informationen zur geplanten oder konkreten Nutzung der Plattform (geschweige denn zu möglichen Einstellungsoptionen) bekommen haben. Häufig sei Antolin plötzlich einfach da gewesen: «lch habe nichts davon gehört, vorher, bevor die Tochter damit ankam» (ANT 1).

Zugleich ist die Bandbreite hinsichtlich der konkreten Involviertheit und Informiertheit von Eltern gross. Es gibt Eltern, die die Plattform eher positiv einschätzen (z. B. bezüglich einer motivierenden Wirkung auf das Leseverhalten ihrer Kinder,

12 Hier spielt auch der regionale Standort der Schule und damit das Lehrpersonen- und Elternklientel eine zentrale Rolle.

13 Dies ist nicht allen Eltern bewusst. So denken Eltern z. T. sie «müssen teilnehmen» (F2B32) oder, dass sie «aus der Nummer nicht rauskommen» (F2B34). 
F1B15/17/21/25; F2B7; F4B38/46) und Eltern, die sich nicht weiter für Antolin interessieren und entsprechend kaum Wissen zur Funktionsweise sowie Basissettings der Plattform haben (F2B20; F3B16; F4B8). Aber es gibt auch solche Eltern, die sich hochgradig in die Nutzung der Plattform einbinden. Dieser «Typ» Eltern unterstützt die bereits skizzierte Wettbewerbslogik, z. B. durch Vergleiche mit anderen Eltern/Kindern und deren Punkteständen, durch Vorlesen oder das gezielte Kaufen bzw. Ausleihen von Antolin-Büchern (F3B1/14). Manche Eltern gehen noch weiter, indem sie selbst aktiv Quizfragen für ihre Kinder beantworten, ihnen bei der Durchführung helfen oder Hörbücher kaufen (ANT 13; F1B16). Bezüglich derartiger Manipulationsstrategien durch Eltern und Kinder (neben den bisher genannten Praktiken u. a. auch Quiz filmen oder Lösungen auf YouTube laden, ANT 2/9/13; F1B44; F2B14) wurde deutlich, dass Lehrpersonen diese Praktiken häufig bewusst sind (siehe auch Abschnitt 4.1): «Bei uns hat eine höchst genervte Klassenlehrerin das Antolin ad acta gelegt, als sie merkte, dass die Mamas die Punkte holten» (F3B13). Andererseits berichtete ein Elternteil von der «Datenverschmutzung» durch sein Kind und dessen Mitschülerinnen und -schüler, da alle Kinder der Klasse ein und dasselbe Konto für die Quizdurchführungen nutzten (ANT 2). Der entsprechenden Lehrperson war dies jedoch unbekannt.

Wie letzteres Beispiel zeigt, steht ein anderer «Typ» Eltern einzelnen Aspekten von Antolin durchaus skeptisch gegenüber. Neben vereinzelten Bedenken hinsichtlich des Themas Datenschutz (ANT 2; F2B34) wird die Validität der Leseleistungserfassung durch die Plattform kritisch hinterfragt (u. a. mit Verweis auf Wettbewerbs- und Manipulationsaktivitäten anderer Eltern - ANT 11; F1B16/24; F2B32/36; F3B1/17). Auch bezüglich möglicher verstärkender sozialer Ungleichheitseffekte zeigen sich einige Eltern sehr reflektiert. Neben dem Bewusstsein für die unterschiedlichen Möglichkeiten des Zugangs zu Büchern (zu Hause/Bücherei, Lebenswelt des Kindes) (ANT 11) wurde dabei z. B. auch das differierende Engagement in den Elternhäusern zum Thema gemacht (ANT 11; F4B22).

Insbesondere wurde aber auch die Quantifizierung von Lesen in Form wettbewerbsorientierter Punktevergaben als bedenklich eingeschätzt (wenn Orden oder Urkunden verliehen werden oder die Punktewerte in die Notengebung mit einfliessen - F1B23; F2B18/32/34; F3B1/14). ${ }^{14}$ Hierbei betonen einige Eltern, dass Lesen Spass machen und Schule ein wettbewerbsfreier Raum sein sollte bzw. durch Benotung und Leistungsdruck ohnehin schon wettbewerbsorientiert (genug) sei (ANT 2; F1B25; F2B14; F3B14). In wenigen Fällen gehen die Bedenken bzw. die Abneigung gegenüber der Plattform so weit, dass Eltern ihren Kindern die Teilnahme an Antolin gänzlich verbieten (ANT 3/13).

Bezüglich der Wirkung von Antolin auf ihre Kinder beobachten Eltern, ähnlich wie einige Lehrpersonen, entsprechend eine starke Wahrnehmungsverschiebung auf

14 Wobei es auch Eltern gibt, die gerade den Wettbewerbs-Faktor von Antolin (in Abgrenzung zu vermeintlicher «Wohlfühlpädagogik», F1B15) als positiv bewerten (ANT 2; F1B15/17). 
Punkte - Effekte der erfolgreichen Regulierung der Wahrnehmung durch das Design der Plattform (siehe Abschnitt 3). So sei es einigen Kindern sehr wichtig, immer wieder zu erzählen, wie viele Punkte sie haben (ANT 1/13; F1B7; F2B7), während andere Kinder die Bewertung der Leseleistung in Form von Punkten «doof» fänden (ANT 3; F1B24). Des Weiteren erwähnten Eltern den Aspekt der Gamifizierung als zentral für ihre Kinder, die die Plattform als «Spiel» nutzen würden (ANT 1/2/10; F3B13/17): «Also darum geht's auf jeden Fall. Er hat [...] bei diesem Programm sozusagen erstmal ausgecheckt, ob man irgendwas Lustiges damit machen kann» (ANT 10). Auch werde dem eigenen Empfinden nach das «Lernprogramm als Ausrede genutzt, um an den Bildschirm zu dürfen» (ebd.). Auf der anderen Seite sei die Plattform auf Dauer nicht «spannend» genug und werde - aufgrund zu weniger spielerischer Elemente schnell langweilig (ANT 10, F2B30).

Insgesamt zeigt sich also auch bei den Elterninterviews und Foreneinträgen eine hochgradige Dynamik der Wirkungen von Antolin zwischen Regulierung und Kontextualisierung, sowohl was die direkten Nutzungspraktiken (= with the platform) als auch was Praktiken mit indirektem Bezug zu Antolin (= beyond the platform) angeht. Schlussfolgerungen, welche sich hieraus sowie aus der vorangegangenen Oberflächenanalyse ergeben, wollen wir im Fazit zusammenfassend diskutieren.

\section{Diskussion und Ausblick}

Mit diesem Beitrag verfolgten wir das Ziel, eine kritische Perspektive auf die komplexe Wirkmächtigkeit von Lernplattformen als Kernbereich zunehmend datengetriebener Schule zu entwickeln. Das zentrale Argument war hierbei, dass Plattformen bestimmte Formen digitaler Bildungswelt erzeugen, strukturieren und in Wert setzen, die entsprechend regulierend auf die ‘analoge Bildungswelt zurückwirken. Und dies gilt nicht nur für 'grosses Plattformen (z. B. G.Suite von Google, Moodle, Schul-Cloud), sondern ebenso für kleine Plattformen wie Antolin, die auf den ersten Blick (nicht jedoch bei näherer Betrachtung) wenige, auf einen einzigen Kompetenzbereich (Lesekompetenz) fokussierte Funktionen für ein einziges Fach (Deutsch) umfassen und daher deutlich harmloser erscheinen mögen.

Mit anderen Worten ist es der übergreifende Prozess- und Strukturzusammenhang wachsender Plattformisierung von Bildung, den es in seinem Facettenreichtum, aber auch in seinen Logiken (u.a. Datafizierung, Personalisierung bzw. Individualisierung als begrenzt flexibilisiertes Agieren im Rahmen vorgegebener Designs ${ }^{15}$ ) zu verstehen und auf den es in der (Medien-) Pädagogik noch deutlich stärker zu reagieren gilt. Das

15 Interessant sind in diesem Zusammenhang Weiterentwicklungen des Programms (insbesondere auf Initiative von Lehrpersonen), um die Plattform noch stärker zu «individualisieren», was jedoch konkret bedeutet, etwa Minuspunkte oder Zeitbegrenzungen schülerbezogen an- und ausschalten zu können oder Lehrpersonen und Schülerinnen und Schüler die Plattformumgebung gestalten zu lassen (z. B. individualisierte Lese-Fleiss-Bilder nach Interesse, Hintergrundmuster) (Westermann 2020b, 4; ANT 9). 
in diesem Beitrag genutzte Konzept der Denkinfrastruktur (Bowker et al. 2019) bietet unseres Erachtens hierfür eine vielversprechende Heuristik; die Methodologien der critical platform studies wiederum fruchtbare methodische Anknüpfungspunkte zur Weiterentwicklung entsprechender Debatten in der deutschsprachigen Forschung zu digitalen Bildungsmedien.

Wie unsere Analyse insgesamt gezeigt hat, erscheint das «disruptive Potential» von Plattformen wie Antolin innerhalb von Lern- und Bildungskontexten mindestens als hochgradig ambivalent, da letztere immer als «[...] interlocking systems of learners, educators, technologies, and broader social contexts, with all kinds of invisible linkages and unexpected consequences» (Reich 2020,9) in Erscheinung treten und es entsprechend immer um die Frage der Sinnzuschreibung innerhalb dieser komplexen Systeme geht (vgl. auch Perrotta 2020). Mit anderen Worten wirken Plattformen, während sich gleichzeitig - wie bei Medien im Allgemeinen - selten vorhersagen lässt, wie genau sich diese Wirkungen praktisch entfalten und inwieweit sie bestehende Bildungspraxis erfolgreich in Frage stellen (siehe auch Fussnote 3).

Interessant ist in diesem Zusammenhang auch die Beobachtung des Bildungsforschers Justin Reich $(2020,132)$, dass digitale Lerntechnologien immer dann viel und gerne in Schule und Unterricht genutzt werden (was bei Antolin definitiv der Fall ist), wenn sie wenig Innovatives (= Unbequem-Verwirrendes) bieten, sondern bekannte instruktive Unterrichtspraktiken möglichst im Digitalen duplizieren. Hierunter fallen beispielsweise vordefinierte Inhalte als zeitlimitierte Aufgaben mit eindeutiger und damit bewertbarer Antwort. Eine tatsächliche Transformation oder gar Revolution traditioneller Bildungspraxis, so formuliert Reich, lässt sich auf diese Weise allerdings kaum erreichen; viel eher droht eine technologische Verschärfung traditioneller Probleme wie etwa Bildungsungleichheit oder prüfungsbezogenes, anstatt kreatives, langfristiges Lernen.

In vielerlei Hinsicht besteht also ein immenser Bedarf an kritischer(-er) Auseinandersetzung, insbesondere bei Lehrpersonen und Eltern. ${ }^{16}$ Gleichzeitig lässt sich nicht alles in einer Verantwortungszuschreibung an die Nutzerinnen und Nutzer (= "gute Nutzung») und damit in eine Frage digitalen Kompetenzerwerbs auflösen, denn selbst wenn Anpassungen in der Nutzung vorgenommen werden (bei Antolin z. B. Ausschalten der Minuspunkte), bleiben die Nutzerinnen und Nutzer dem Design und damit der digitalen Inwertsetzung von Bildung als solches in der Regel ausgeliefert. Für die politische und erziehungswissenschaftliche Förderung digitaler Kompetenz im Sinne von critical data oder critical platform literacy muss dies entsprechend bedeuten, die bewusste Nicht-Nutzung von Plattformen in pädagogisch legitime Entscheidungsszenarien systematisch einzubeziehen. ${ }^{17}$

16 Siehe hierzu auch Initiativen wie https://unblackthebox.org.

17 Wir danken den anonymen Gutachtenden für ihre konstruktive Kritik und wertvollen Hinweise. 


\section{Literatur}

Allert, Heidrun. 2020. «Plattformökonomie und Entstaatlichung: familienorientiert, ortsunabhängig und \#freilernend». In Digitalisierung-Subjekt-Bildung: Kritische Betrachtungen der digitalen Transformation, herausgegeben von Valentin Dander, Patrick Bettinger, Estella Ferraro, Christian Leineweber und Klaus Rummler, 183-212. Verlag Barbara Budrich. https://doi.org/10.2307/j.ctvvb7n3h.13.

Allert, Heidrun, und Christoph Richter. 2020. «Learning Analytics: subversive, regulierende und transaktionale Praktiken». In Big Data, Datafizierung und digitale Artefakte, herausgegeben von Stefan Iske, Johannes Fromme, Dan Verständig und Katrin Wilde, 15-35. Medienbildung und Gesellschaft. Wiesbaden: Springer VS Verlag für Sozialwissenschaften. https:// doi.org/10.1007/978-3-658-28398-8_2.

Ang, len. 1995. Living room wars. Rethinking media audiences for a postmodern world. London, New York: Routledge.

Aßmann, Sandra, Nils Brüggen, Valentin Dander, Harald Gapski, Gerda Sieben, Angela Tillmann, und Isabel Zorn. 2017. «Digitale Datenerhebung und -verwertung als Herausforderung für Medienbildung und Gesellschaft. Ein medienpädagogisches Diskussionspapier zu Big Data und Data Analytics». In Medienpädagogik. Eine Standortbestimmung, herausgegeben von Christine Trültzsch-Wijnen, 183-192. Baden-Baden: Nomos Verlagsgesellschaft. https://doi.org/10.5771/9783845279718-183.

BMBF (Bundesministerium für Bildung und Forschung). 2016. «Bildungsoffensive für die digitale Wissensgesellschaft. Strategie des Bundesministeriums für Bildung und Forschung». Herausgegeben von Bundesministerium für Bildung und Forschung. Oktober, 2016. https://www.bmbf.de/files/Bildungsoffensive_fuer_die_digitale_Wissensgesellschaft.pdf

Bowker, Geoffrey C., Julia Elyachar, Martin Kornberger, Andrea Mennicken, Peter Miller, Joanne Randa Nucho, und Neil Pollock. 2019. «Introduction to Thinking Infrastructures». In Thinking Infrastructures, herausgegeben von Martin Kornberger, Geoffrey C. Bowker, Julia Elyachar, Andrea Mennicken, Peter Miller, Joanne Randa Nucho, und Neil Pollock. Research in the Sociology of Organizations 62: 1-13. Emerald Publishing Limited. https://doi. org/10.1108/S0733-558X20190000062001.

Breiter, Andreas, und Angelina Lange. 2019. «Die digitale Schule und Schulverwaltung». In Handbuch Digitale Verwaltung, herausgegeben von Hans-Henning Lühr, Roland Jabkowski und Sabine Smentek. 1. Auflage, 330-342. Wiesbaden: Kommunal- und Schul-Verlag Wiesbaden.

Bucher, Taina. 2018. If... then: Algorithmic power and politics. Oxford: Oxford University Press. https://doi.org/10.1093/oso/9780190493028.001.0001.

Decuypere, Mathias. 2019. «Researching educational apps: Ecologies, technologies, subjectivities and learning regimes». Learning, Media and Technology 44 (4): 414-429. https://doi.org /10.1080/17439884.2019.1667824.

Decuypere, Mathias. 2021. «The Topologies of Data Practices: a Methodological Introduction». Journal of New Approaches in Educational Research 10(1): 1-17. https://doi.org/10.7821/ naer.2021.1.650. 
Decuypere, Mathias, und Paolo Landri. 2020. «Governing by visual shapes: university rankings, digital education platforms and cosmologies of higher education». Critical Studies in Education, 1-17. https://doi.org/10.1080/17508487.2020.1720760.

Dieter, Michael, Carolin Gerlitz, Anne Helmond, Nathaniel Tkacz, Fernando van der Vlist, und Esther Weltevrede. 2018. "Store, interface, package, connection. Methods and propositions for multi-situated app studies». SFB 1187 Medien der Kooperation - Working Paper Series, Band 4 (2018): 1-16. https://www001.zimt.uni-siegen.de/ojs/index.php/wps1187/ article/view/29.

Endlein, Verena. 2009. «Beeinflusst Antolin das Leseverhalten? Eine qualitative Studie». Hausarbeit zur Diplomprüfung, Hochschule für Angewandte Wissenschaften Hamburg.

Fischer, Felix. 2019. «Imagination by Design: Imagineered Agency und die Frage nach Selbstbestimmung in designten digitalen Umgebungen». MedienPädagogik: Zeitschrift für Theorie Und Praxis Der Medienbildung 36 (Teilhabe):1-17. https://doi.org/10.21240/ mpaed/36/2019.11.09.X.

Friedrichs-Liesenkötter, Henrike, Lara Gerhardts, Anna-Maria Kamin, und Sonja Kröger, Hrsg. 2020. Medienpädagogik als Schlüsseldisziplin in einer mediatisierten Welt. Perspektiven aus Theorie, Empirie und Praxis. Bd. 37. Themenhefte. Zürich: Zeitschrift MedienPädagogik. https://doi.org/10.21240/mpaed/37.X.

Gillespie, Tarleton. 2010. «The politics of 'platforms'». New Media \& Society 12 (3): 347-364. https://doi.org/10.1177/1461444809342738.

Grundschule Hönebach. o.J. «Unsere besten Antolinsammler». Zugriff 9. Dezember 2020. https://grundschule-hönebach.de/index.php/antolin-auszeichnungen.

Gulson, Kalervo N., Steven Lewis, Bob Lingard, Christopher Lubienski, Keita Takayama, und P. Taylor Webb. 2017. "Policy mobilities and methodology. A proposition for inventive methods in education policy studies». Critical Studies in Education 58 (2): 224-241. https://doi. org/10.1080/17508487.2017.1288150.

Hartong, Sigrid. 2020. «The Power of Relation-Making: Insights into the Production and Operation of Digital School Performance Platforms in US State Education Agencies». Critical Studies in Education, 1-16. https://doi.org/10.1080/17508487.2020.1749861.

Hartong, Sigrid, und Annina Förschler. 2019. «Opening the black box of data-based school monitoring: data infrastructures, flows and practices in state education agencies». Big Data \& Society 6 (1): 1-12. https://doi.org/10.1177/2053951719853311.

Henningsen, Lisa. 2019. «Leseförderung durch Antolin? Was Kinder denken». Masterarbeit, Universität Hamburg.

Iliadis, Andrew, und Federica Russo. 2016. «Critical data studies: An introduction». Big Data \& Society 3 (2). https://doi.org/10.1177/2053951716674238.

Iske, Stefan, Johannes Fromme, Dan Verständig, und Katrin Wilde, Hrsg. 2020. Big Data, Datafizierung und digitale Artefakte. Wiesbaden: Springer VS. https://doi.org/10.1007/978-3658-28398-8.

Jarke, Juliane, und Andreas Breiter. 2019. «Editorial: The datafication of education». Learning, Media and Technology, 44 (1): 1-6. https://doi.org/10.1080/17439884.2019.1573833. 
Jörissen, Benjamin. 2018. Bildung und Design - Pädagogik als Subjektdesign. https://joerissen. name/kongresse-tagungen/bildung-und-design/.

Jörissen, Benjamin, und Dan Verständig. 2017. «Code, Software und Subjekt». In Das umkämpfte Netz, herausgegeben von Ralf Biermann und Dan Verständig, 37-50. Wiesbaden: Springer VS. https://doi.org/10.1007/978-3-658-15011-2_3.

Jornitz, Sieglinde, und Christoph Leser. 2018. «Mit Antolin punkten oder: Wie sich mit dem Leseförderprogramm der Bock zum Gärtner macht». Pädagogische Korrespondenz (57): 55 73. https://doi.org/10.25656/01:21100.

Kitchin, Rob, und Tracey P. Lauriault. 2014. «Towards critical data studies: Charting and unpacking data assemblages and their work». http://mural.maynoothuniversity.ie/5683/1/KitchinLauriault_CriticalDataStudies_ProgrammableCity_WorkingPaper2_SSRN-id2474112. pdf.

Kleiner, Brigitte. 2009. «Lesebuchfreier Leseunterricht mit „Antolin“ - nachhaltiger und kreativer Leseunterricht». In Kreativität und Innovationskompetenz im digitalen Netz. Wie kommt das „Neue“ mit Hilfe von Internettechnologien in die Welt?, herausgegeben von Veronika Hornung-Prähauser und Michaela Luckmann, 274-279. Sammlung von ausgewählten Fachund Praxisbeiträgen der 5. EduMedia Fachtagung 2009. Salzburg, 04.-05. Mai 2009. Salzburg: Research Forschungsgesellschaft $\mathrm{mbH}$.

KMK (Kultusministerkonferenz). 2016. «Bildung in der digitalen Welt. Strategie der Kultusministerkonferenz». Herausgegeben von Kultusministerkonferenz. Dezember, 2016. https:// www.kmk.org/fileadmin/Dateien/pdf/PresseUndAktuelles/2017/Strategie_neu_2017_datum_1.pdf.

Landri, Paolo. 2018. Digital Governance of Education. Technology, Standards and Europeanization of Education. London: Bloomsbury Academic.

Manolev, Jamie, Anna Sullivan, und Roger Slee. 2019. «The datafication of discipline: ClassDojo, surveillance and a performative classroom culture». Learning, Media and Technology 44 (1): 36-51. https://doi.org/10.1080/17439884.2018.1558237.

Martin, Lauren, und Anna J. Secor. 2014. «Towards a post-mathematical topology». Progress in Human Geography 38 (3): 420-438. https://doi.org/10.1177/0309132513508209.

Perrotta, Carlo. 2020. «Programming the platform university: Learning analytics and predictive infrastructures in higher education». Research in Education. https://doi. org/10.1177/0034523720965623.

Ratner, Helene, und Christopher Gad. 2019. «Data Warehousing Organization: Infrastructural Experimentation with Educational Governance». Organization 26 (4): 537-552. https://doi. org/10.1177/1350508418808233.

Reich, Justin. 2020. Failure to disrupt. Why technology alone can't transform education. Harvard: Harvard University Press.

Ruppert, Evelyn, Engin Isin, und Didier Bigo. 2017. «Data Politics». Big Data \& Society. https:// doi.org/10.1177/2053951717717749. 
Viertel, Michael, Yvonne Ehrenspeck-Kolasa, und Anke Spies. 2017. «Digitale Leseförderung an Grundschulen zwischen Anspruch und Wirklichkeit. Eine Untersuchung zur Nutzung und Bewertung der web-basierten Leseförderung >Antolin` durch Grundschullehrkräfte in Niedersachen (NuBeAn)». In Jahrbuch Medienpädagogik 13, herausgegeben von Kerstin Mayrberger, Johannes Fromme, Petra Grell, und Theo Hug, 151-164. Wiesbaden: Springer VS Verlag für Sozialwissenschaften. https://doi.org/10.1007/978-3-658-16432-4_10.

Westermann (Westermann Bildungsmedien Verlag GmbH). 2017. «Nutzerhandbuch». Veröffentlicht auf www.antolin.de. https://antolin.westermann.de/all/downloads/nutzerhandbuch.pdf.

Westermann. 2020a. «Große Nachfrage nach Westermann-Angeboten für das Lernen zu Hause». Bildungsklick, Bundesweite Pressemitteilung, 24. März 2020. https://bildungsklick.de/ schule/detail/grosse-nachfrage-nach-westermann-angeboten-fuer-das-lernen-zu-hause.

Westermann. 2020b. «Antolin. Mit Lesen punkten! Das Programm zur Leseförderung im neuen Gewand: frisch, freundlich, farbenfroh und optimiert». Prospekt veröffentlicht auf www. antolin.de, 2020. https://antolin.westermann.de/all/downloads/antolin_prospekt.pdf.

Westermann. 2020c «Das Online-Programm zur Leseförderung von Klasse 1 bis 10. Präsentation zur Einführung». Powerpoint-Präsentation veröffentlicht auf www.antolin.de. https:// antolin.westermann.de/all/downloads/antolin_ppt.pdf.

Westermann o.J.a. «Mit Antolin arbeiten». Veröffentlicht auf www.antolin.westermann.de. Zugriff 09. Dezember 2020. https://antolin.westermann.de/all/info/mit-antolin-arbeiten.jsp.

Westermann. o.J.b. «Quizfragen - der Kern von Antolin». Veröffentlicht auf www.antolin.de. Zugriff 09. Dezember 2020. https://antolin.westermann.de/all/info/quizfragen_-_der_ kern.jsp.

Westermann. o.J.c. «Lizenzen». Veröffentlicht auf www.antolin.de. Zugriff 09. Dezember 2020. https://antolin.westermann.de/all/lizenzen.jsp.

Westermann. o.J.d. «So funktioniert Antolin». Veröffentlich auf www. antolin.westermann.de. Zugriff 09. Dezember 2020. https://antolin.westermann.de/all/info/so_funktioniert_antolin.jsp.

Westermann. o.J.e. «Auf dem Weg zur Leseschule». Veröffentlicht auf www.antolin.westermann.de. Zugriff 09. Dezember 2020. https://antolin.westermann.de/nl/nl_06_11_nov/ nl_06_11_nov_leseschule.jsp.

Westermann o.J.f. «Mitarbeit der Eltern». Veröffentlicht auf www.antolin.westermann.de. Zugriff 09. Dezember 2020. https://antolin.westermann.de/all/info/mitarbeit_der_eltern.jsp

Williamson, Ben. 2017. Big data in education. The digital future of learning, policy and practice. London: SAGE Publications.

Zorn, Isabel. 2011. «Zur Notwendigkeit der Bestimmung einer auf digitale Medien fokussierten Medienkompetenz und Medienbildung». MedienPädagogik: Zeitschrift für Theorie Und Praxis Der Medienbildung 20 (Medienbildung - Medienkompetenz): 175-209. https://doi. org/10.21240/mpaed/20/2011.09.19.X.

Zschocher, Andrea. 2020. «Antolin Punkte: Lernen Kindern durch die App wirklich besser lesen?». Veröffentlicht auf www.familie.de, 20. August 2020. https://www.familie.de/schulkind/antolin-punkte-lernen-kindern-durch-die-app-wirklich-besser-lesen/. 